\title{
Lattice type of fracture model for concrete
}

\author{
J.X. Liu *, S.C. Deng, J. Zhang, N.G. Liang \\ State Key Laboratory of Nonlinear Mechanics, Institute of Mechanics, Chinese Academy of Sciences, 100080 Beijing, China
}

Available online 21 September 2007

\begin{abstract}
Concrete is usually described as a three-phase material, where matrix, aggregate and interface zones are distinguished. The beam lattice model has been applied widely by many investigators to simulate fracture processes in concrete. Due to the extremely large computational effort, however, the beam lattice model faces practical difficulties. In our investigation, a new lattice called generalized beam (GB) lattice is developed to reduce computational effort. Numerical experiments conducted on a panel subjected to uniaxial tension show that the GB lattice model can reproduce the load-displacement curves and crack patterns in agreement to what are observed in tests. Moreover, the effects of the particle overlay on the fracture process are discussed in detail.
\end{abstract}

(C) 2007 Elsevier Ltd. All rights reserved.

Keywords: Fracture; Generalized beam (GB) lattice model; Concrete; Particle overlay; Computational effort

\section{Introduction}

Analysis of fracture processes in concrete is a timely research topic. The matter is highly complicated due to the heterogeneous structure of the material over many different length scales. From the nanometer to the micrometer scale the structure of cement appears to be very heterogeneous. Even at the smallest level, i.e. the nanometer scale, the structure of hydrated cement appears elusive [1]. Scaling upward towards the micrometer or millimeter level will bring new elements in the structure, namely the sand and aggregate particles. Sand and aggregate have typical mineralogical compositions, which makes it interesting to study them at the microlevel as well. For concrete, however, the interface zone between aggregate and bulk cement paste appears to be the most important [2]. Therefore, concrete is usually considered as a three-phase material, i.e. matrix, aggregate and interface zones are distinguished $[3,4]$.

Two main approaches can be differentiated to deal with fracture processes in concrete. The classical is the approach where the material structure is excluded, and phenomenological relations between global stress and strain are determined with the help of experiments. In the second approach parameters of the material structure are included directly in the formulation. In this so-called microapproach it is important to decide to what level structure information is incorporated. The second approach can also be regarded as a more

\footnotetext{
* Corresponding author. Tel.: +86 1062545533 3182; fax: +86 1062579511.

E-mail address: liujx@lnm.imech.ac.cn (J.X. Liu).
} 
physical-based approach since detailed information about the fracture process in relation to the material structure is obtained [3].

There may be two different methods to incorporate the material structure. In the first method, the details about the structure can be measured and translated directly into a finite element mesh. Details about granular size distribution, interface geometry and so on are used directly [4]. As a result distinct regions of varying stiffness and strength will be present in the mesh. In the other method, the structure information is translated into a statistical distribution that is subsequently used to assign strength and stiffness properties to different elements in a finite element mesh [3]. For regular lattices, it was shown [3,5] that although the effects of material structure mimicked by assigning statistical distribution of properties according to Weibull or Gauss distribution save much computation effort, more realistic results are obtained by projecting directly the material structure on to the lattice [4]. However, in order to include the interface zones directly, the mesh size, i.e. the span of beams, must be limited to the same order of magnitude as the interfacial thickness. As a result, this leads to practical difficulties, due to the extremely large computational effort.

In order to solve the problem of computational effort in the existing lattice models, the development of a new lattice-type fracture model called GB lattice model is the main objective of this paper. Obviously, according to the basic idea of finite element method, the mesh size has to be of the same order of magnitude as the meso-level characteristic length in order to observe the detailed response at this level. This investigation shows that, however, the three-phase material structure can be projected directly on top of a regular triangular GB lattice whose mesh size can be even larger than the maximum aggregate. The key technique is the development of a kind of two-node and three-phase elements, which are different from beam elements, truss elements or springs commonly used in the existing lattice models. Thus, the three-phase element is called generalized beam (GB) element, and the corresponding lattice is called GB lattice. A GB element is composed of three beams. Every beam in GB elements can be a matrix beam, interface beam or aggregate beam. In the beam lattice 10100 nodes are usually needed to simulate an aggregate, but just a single node is required to model an aggregate in the GB lattice. As a result, computational effort is extremely reduced when the GB lattice model is adopted.

In a GB element, the geometrical and material parameters of each beam are calibrated independently. Like the corresponding work in [4], aggregate beams and matrix beams are taken as Timoshenko beams and their parameters are calibrated. Whilst interface beams are described by (A1) in Appendix A [6], and real interfacial properties are assigned to them.

For ideally brittle lattices, as long as a strength criterion is adopted for failure of the elements, a typical procedure for the lattice simulation is as follows [7-9]. The load is applied gradually and linear elastic analysis is performed until the element with the highest stress-to-strength ratio reaches the prescribed threshold value [10]. It is then eliminated and a new analysis is performed after updating the stiffness matrix while the applied load is kept unchanged to check whether another element will fail. If no more elements fail, the calculation is restarted from zero loads again until the complete failure of the specimen $[3,4]$.

It is interesting to better understand the details of the fracture process in concrete. Firstly, micro-cracking was caused in the interface between matrix and aggregates by stress concentrations $[4,10]$. Once the peak-load was reached cracks localized in the matrix [10], as was also shown by means of photo-elastic coating [11]. Directly following the peak load, a steep drop in load was commonly observed [4]. Usually at several places along the macro-crack path, it was found that it deviated from a straight path [12]. The ductility of specimens was also discussed frequently $[3,4,10,11]$. Here, ductility is defined as the ratio between the energy consumed during the fracture process after and before the peak $[4,10]$.

In order to solve the problem of brittleness in load-displacement relations obtained from 2D lattice analyses, Lilliu et al. [10] developed a 3D lattice model. This 3D lattice, however, was hampered by a substantial increase of computational effort. Moreover, the specimen simulated in [10] was so small that it could not be a representative of the overall material behavior, since boundary effects was expected. Therefore, the $2 \mathrm{D}$ simulation may be a more proper choice for qualitative analyses of fracture processes.

In this paper, the effects of the computer-generated particle overlay [3,4] on the fracture process are discussed in detail. To do so, two groups of numerical experiments are employed. In Group I, all the cases have a fixed particle overlay and different material properties. In Group II, all the cases have the same material properties and different particle overlays, and the number of particles of each size is kept to be unchanged with particle overlay varying. 


\section{The geometry of the GB lattice model}

At first, the creation of the beam lattice model is recalled. To obtain more realistic results, the material structure is projected directly on to the lattice $[3,5]$. In Fig. 1, if both ends of a beam fall in the matrix (aggregate) phase, it is assigned the equivalent matrix (aggregate) properties. If however one of its ends is in the matrix phase and the other is in the aggregate phase, then it is assigned the equivalent interface properties. The relationship between the properties of a continuum phase and its representative beams in the lattice is obtained by the equivalence of strain energy stored in a unit cell under constant strains $[4,7,13]$.

Like the above beam lattice, it is also in a very straightforward way that the GB lattice corresponds to the material structure. In Fig. 2a, an aggregate is projected on to a matrix GB lattice, with its center lying on node $i$. The six elements around node $i$ are partly overlain by the aggregate. These parts are assigned the equivalent aggregate properties. The interface is simplified as a thin layer with a rectangular cross-section clinging to the aggregate. The parts of the six elements overlain by the interface are assigned the interfacial properties. Finally, the GB lattice including the particle structure is shown in Fig. 2 b.

The GB element is apparently a two-node and three-phase element. Every phase of the element is represented by a beam of corresponding (equivalent) properties. For example, in Fig. 2b, the six GB elements around node $i$ are all composed of an aggregate beam, an interface beam and a matrix beam. However, it can be imagined that the GB element which is not overlain by any aggregate is actually one-phase, i.e. matrix phase. Nevertheless, in order to make up the deficiency of the relatively coarser GB lattice, the one-phase GB elements are divided into three matrix beams of the same span. When a GB element is overlain by two aggregates, the element is actually composed of two aggregate beams, two interface beams and one matrix beam. For simplification, this kind of elements is considered to be composed of one interface beam and two aggregate beams of the same span. In a word, all GB elements are composed of three beams.
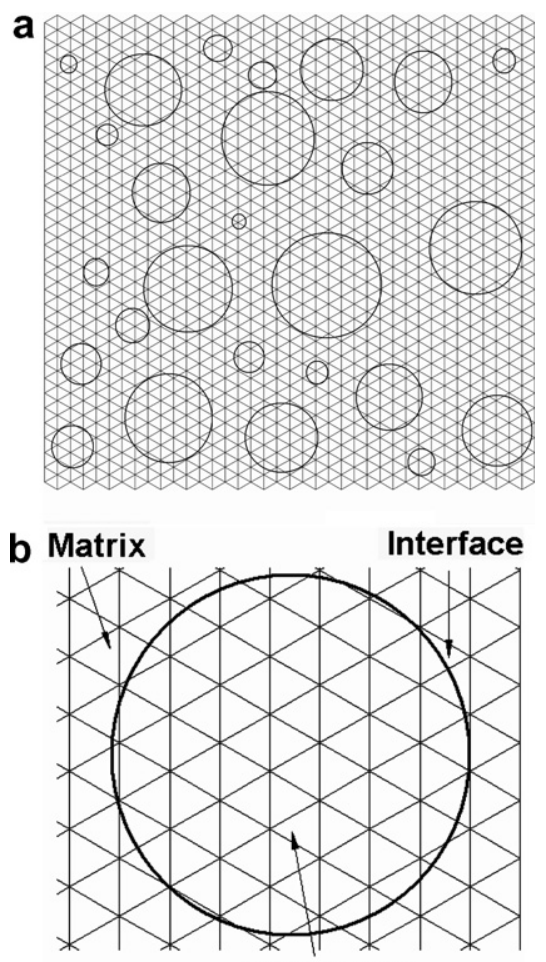

Aggregate

Fig. 1. Particle structure modeled by the beam lattice model: (a) general particle structure; (b) definition of matrix, interface and aggregate element. 

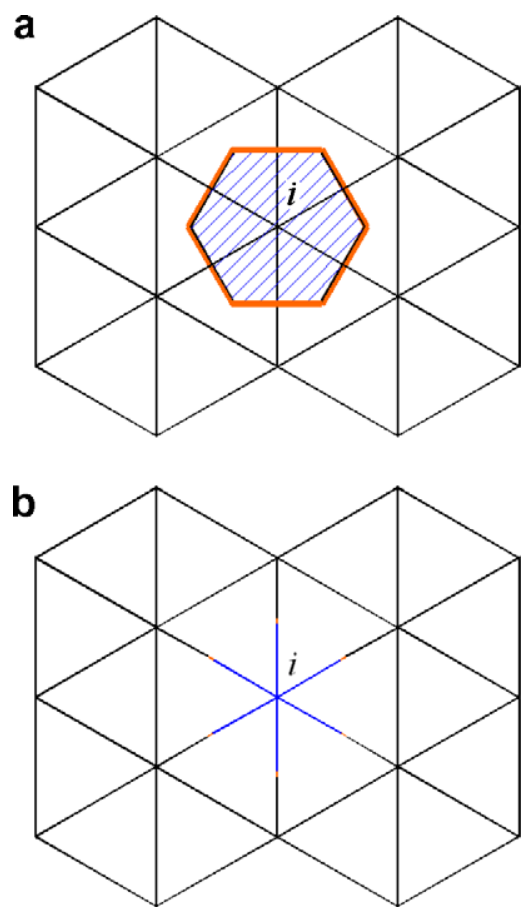

Fig. 2. A GB lattice including particle structure: (a) the inclusion of the aggregate; and (b) its corresponding GB lattice.

Found when Fig. 1b is compared with Fig. 2b, an aggregate usually covers 10-100 nodes in the beam lattice, but an aggregate just overlays a single node in the GB lattice. As a result, computational effort is extremely reduced in the GB lattice model.

If three beams in each GB element are assumed to cling firmly to each other and deform together without sliding, the displacements of two ends of the middle beam in the GB element are completely determined by the displacements of the two nodes of the GB element, which will be discussed in detail in Section 5. Then, the degrees of freedom of two ends of the middle beam are not necessarily included in the discrete system of equations of equilibrium, with the result that computational effort is further reduced.

\section{Stiffness matrices of matrix, aggregate and interface beams}

Matrix beams and aggregate beams are described by Timoshenko beam theory. However, Timoshenko beam theory cannot describe interface beams, because the aspect ratio of interface beams is usually too high. In this paper, interface beams are described by (A1) in Appendix A of Bolander et al. [6]. These two kinds of stiffness matrices have the following common expression (Fig. 3):

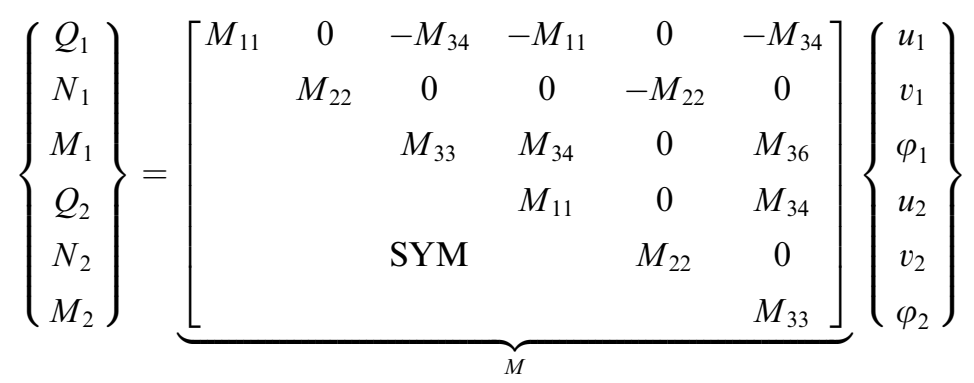

where $\mathbf{F}_{12}=\left\{Q_{1} N_{1} M_{1} Q_{2} N_{2} M_{2}\right\}^{\mathrm{T}}$ and $\mathbf{u}_{12}=\left\{u_{1} v_{1} \varphi_{1} u_{2} v_{2} \varphi_{2}\right\}^{\mathrm{T}}$ are the generalized force vector and the generalized displacement vector, respectively. 


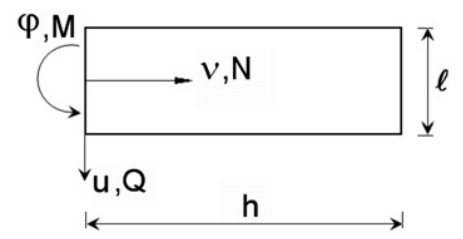

Fig. 3. Kinematics and statics of a beam.

For a Timoshenko beam, the independent elements in (1) can be expressed in the form [14,15]

$$
M_{11}=\frac{12 E^{(b)} I}{(1+b) h^{3}}, \quad M_{34}=\frac{6 E^{(b)} I}{(1+b) h^{2}}, \quad M_{22}=\frac{E^{(b)} A}{h}, \quad M_{33}=\frac{E^{(b)} I(4+b)}{h(1+b)}, \quad M_{36}=\frac{E^{(b)} I(2-b)}{h(1+b)}
$$

where $E^{(b)}$ is the Young's Modulus; $t^{(b)}, h$ and $l$ are respectively the thickness, the span and the height of the Timoshenko beam; $A=t^{(b)} l$ is the cross-section area; $I=t^{(b)} l^{3} / 12$ is the moment of inertia; $b=6 E^{(b)} l^{2} / 5 G^{(b)} h^{2}$ is the dimensionless parameter in Timoshenko beam theory; $G^{(b)}=E^{(b)} / 2\left(1+v^{(b)}\right)$ is the modulus of rigidity, where $v^{(b)}$ is the Poisson's ratio. By comparing (A1) in Appendix A of [6] with (1), the independent elements in (1) can be expressed in the form

$$
M_{11}=\frac{G^{(b)} A}{h}, \quad M_{34}=\frac{G^{(b)} A}{2}, \quad M_{22}=\frac{E^{(b) \prime} A}{h}, \quad M_{33}=\frac{G^{(b)} A h}{4}+\frac{E^{(b) \prime} I}{h}, \quad M_{36}=\frac{G^{(b)} A h}{4}-\frac{E^{(b) \prime} I}{h}
$$

where $E^{(b) \prime}=E^{(b)} /\left[1-\left(v^{(b)}\right)^{2}\right]$.

In the following sections, a superscript is added to $\mathbf{M}$ in order to declare the material property, i.e. $\mathbf{M}^{\mathrm{m}}, \mathbf{M}^{\mathrm{a}}$ and $\mathbf{M}^{\mathrm{i}}$ are respectively the stiffness matrix of matrix, aggregate and interface beams. When an aggregate is very small, the interface beams clinging to the aggregate have a relatively lower aspect ratio. In this case, it is more proper to take these interface beams as Timoshenko beams. However, in the present investigation, for simplification, all interface-phase beams adopt the stiffness matrix in (3).

\section{Parameter calibration of the regular triangular GB lattice}

Spans of three beams of each GB element are determined as soon as the particle overlay [3] is projected on top of the GB lattice. To an interface beam, the properties of interface material themselves instead of its equivalent properties are assigned. Moreover, the depth of the interface beam can be calculated in the form

$$
l= \begin{cases}2 d_{i} / \sqrt{3} & \left(d_{i} \leqslant L / 2\right) \\ 2\left(L-d_{i}\right) / \sqrt{3} & \left(L / 2 \leqslant d_{i} \leqslant L\right)\end{cases}
$$

where $L$ is the length of the GB element, and $d_{i}$ denotes the distance between node $i$ and the middle point of the interface beam along its span-direction, in the GB element $i-j$.

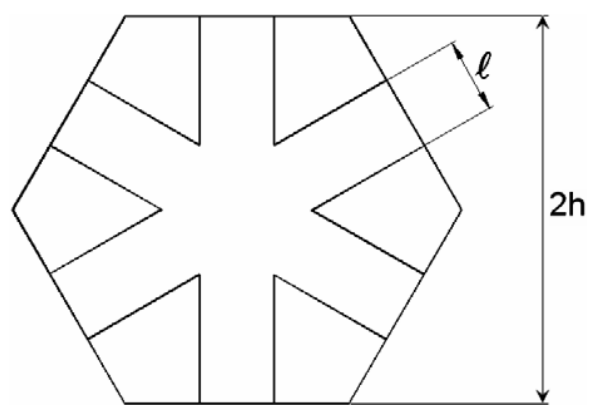

Fig. 4. A triangular matrix/aggregate GB lattice with a hexagonal unit cell. 
Then we introduce how to calculate the geometrical and material properties of both matrix and aggregate beams. The basic idea is based on the equivalence of strain energy stored in a unit cell (Fig. 4) of a lattice with its continuum counterpart under constant strains

$$
U_{\text {cell }}=U_{\text {continuum }}
$$

The detailed process has been presented in [4] to obtain the relationship between the triangular Timoshenko beam lattice and its micropolar continuum equivalent. In the present investigation, the result of Karihaloo et al. [4] is used directly in our parameter system (Fig. 4):

$$
\frac{E}{E^{(b)}}=\sqrt{3}\left(\frac{l}{h}\right) \frac{t^{(b)}}{t} \frac{4+\left(\frac{l}{h}\right)^{2} \frac{1}{1+b}}{12+\left(\frac{l}{h}\right)^{2} \frac{1}{1+b}}, \quad v=\frac{4-\left(\frac{l}{h}\right)^{2} \frac{1}{1+b}}{12+\left(\frac{l}{h}\right)^{2} \frac{1}{1+b}}
$$

where $E^{(b)}$ and $E$ are the Young's modulus of the Timoshenko beams and its continuum equivalent. $h$ and $l$ are the span and depth of the Timoshenko beam. $t^{(b)}$ and $t$ are the thickness of the Timoshenko beam and its continuum equivalent. Moreover,

$$
b=\frac{12+11 v^{(b)}}{20}\left(\frac{l}{h}\right)^{2}
$$

The second equation of (6) can be rewritten in the form

$$
b=\frac{1+v}{4(1-3 v)}\left(\frac{l}{h}\right)^{2}-1
$$

Substitution of (7) into the second equation of (6) yields the result

$$
v^{(b)}=\frac{5(1+v)}{11(1-3 v)}-\frac{20}{11}\left(\frac{l}{h}\right)^{-2}-\frac{12}{11}
$$

Substitution of (8) into the first equation of (6) yields the result

$$
E^{(b)}=\frac{2}{\sqrt{3}}\left(\frac{l}{h}\right)^{-1} \frac{t}{t^{(b)}} \frac{E}{1-v}
$$

In this investigation, all matrix beams and aggregate beams have rectangular cross-sections, the same aspect ratio $l / h=1$, and the same thickness $t^{(b)}=t$. The Poisson's ratio of both matrix and aggregate is $11 / 40$. Then, from (9) and (10), their equivalent material properties $v^{(b)}$ and $E^{(b)}$ respectively become

$$
\begin{aligned}
& v^{(b)}=\frac{31}{77} \\
& E^{(b)}=\frac{80}{29 \sqrt{3}} E
\end{aligned}
$$

\section{The stiffness matrix of a GB element}

This section is going to answer how to determine the stiffness matrix of a GB element from its three beams. For the sake of simplification and without loss of generality, a GB element composed of one aggregate beam, one interface beam and one matrix beam is under investigation (Fig. 5).

In the GB element $i-j$ (Fig. 5a), when external generalized forces are applied on node $i$ and $j$, the relationship between the generalized nodal forces and the generalized nodal displacements can be expressed in the form

$$
\begin{aligned}
\mathbf{F}_{i j} & =K \mathbf{u}_{i j} \\
\mathbf{F}_{i j} & =\left\{\begin{array}{llllll}
Q_{i} & N_{i} & M_{i} & Q_{j} & N_{j} & M_{j}
\end{array}\right\}^{\mathrm{T}} \\
\mathbf{u}_{i j} & =\left\{\begin{array}{llllll}
u_{i} & v_{i} & \varphi_{i} & u_{j} & v_{j} & \varphi_{j}
\end{array}\right\}^{\mathrm{T}}
\end{aligned}
$$

where $\mathbf{K}$, a symmetric matrix, denotes the stiffness matrix of the GB element. 

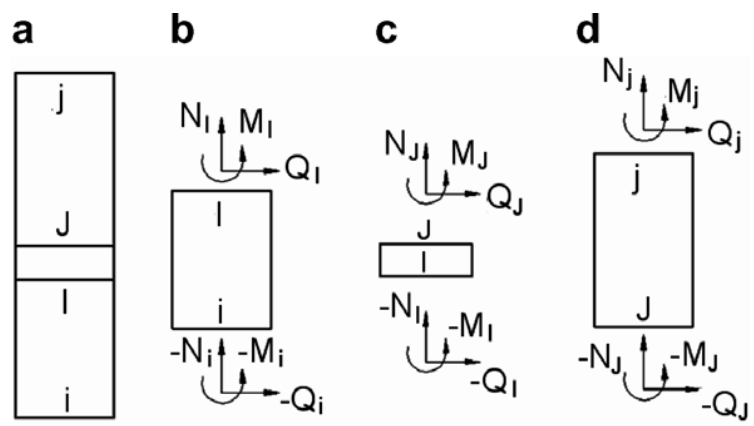

Fig. 5. A GB element composed of an aggregate beam, an interface beam and a matrix beam: (a) the GB element; (b) the aggregate beam; (c) the interface beam; (d) the matrix beam.

Analogously, for the aggregate beam $i-I$ (Fig. 5b), the interface beam $I-J$ (Fig. 5c), and the matrix beam $J-j$ (Fig. 5d), the relationships between the kinematics and statics can be expressed in the forms, respectively

$$
\begin{aligned}
& i-I: \\
& \mathbf{F}_{i I}=\mathbf{M}^{\mathrm{a}} \mathbf{u}_{i I} \\
& \mathbf{F}_{i I}=\left\{\begin{array}{llllll}
Q_{i} & N_{i} & M_{i} & Q_{I} & N_{I} & M_{I}
\end{array}\right\}^{\mathrm{T}} \\
& \mathbf{u}_{i I}=\left\{\begin{array}{llllll}
u_{i} & v_{i} & \varphi_{i} & u_{I} & v_{I} & \varphi_{I}
\end{array}\right\}^{\mathrm{T}} \\
& I-J: \\
& \mathbf{F}_{I J}=\mathbf{M}^{\mathrm{i} \mathbf{u}_{I J}} \\
& \mathbf{F}_{I J}=\left\{\begin{array}{llllll}
-Q_{I} & -N_{I} & -M_{I} & Q_{J} & N_{J} & M_{J}
\end{array}\right\}^{\mathrm{T}} \\
& \mathbf{u}_{I J}=\left\{\begin{array}{lllllll}
u_{I} & v_{I} & \varphi_{I} & u_{J} & v_{J} & \varphi_{J}
\end{array}\right\}^{\mathrm{T}} \\
& J-j: \\
& \mathbf{F}_{J j}=\mathbf{M}^{\mathrm{m}} \mathbf{u}_{J j} \\
& \mathbf{F}_{J j}=\left\{\begin{array}{llllll}
-Q_{J} & -N_{J} & -M_{J} & Q_{j} & N_{j} & M_{j}
\end{array}\right\}^{\mathrm{T}} \\
& \mathbf{u}_{J j}=\left\{\begin{array}{llllll}
u_{J} & v_{J} & \varphi_{J} & u_{j} & v_{j} & \varphi_{j}
\end{array}\right\}^{\mathrm{T}}
\end{aligned}
$$

Table 1

Particle distribution

\begin{tabular}{llllll}
\hline Size $(\mathrm{mm})$ & $0.4 L$ & $0.6 L$ & $0.8 L$ & $1.0 L$ & $1.2 L$ \\
Number & 161 & 67 & 86 & 92 & 93 \\
\hline
\end{tabular}

$L=\sqrt{3} / 10 \mathrm{~cm}$ is the length of the GB elements.

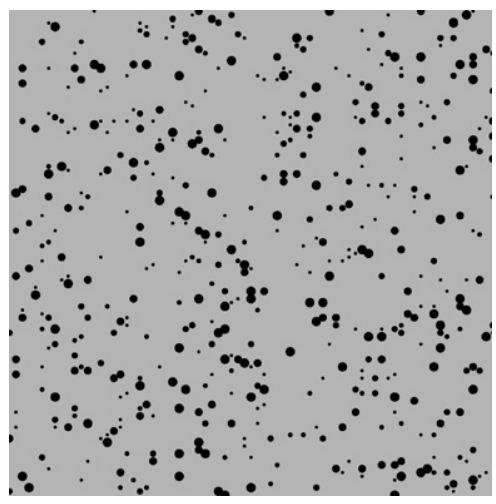

Fig. 6. The computer-generated particle overlay of Group I. 
In consideration of the equilibrium conditions at $I$ and $J$, a system of algebraic equations expressed in the generalized displacement vector $u_{I J}$ can be obtained when $u_{i j}$ are assumed to be known

$$
\underbrace{\left[\begin{array}{cc}
\mathbf{M}_{\mathrm{III}}^{\mathrm{a}}+\mathbf{M}_{\mathrm{I}}^{\mathrm{i}} & \mathbf{M}_{\mathrm{II}}^{\mathrm{i}} \\
\left(\mathbf{M}_{\mathrm{II}}^{\mathrm{i}}\right)^{\mathrm{T}} & \mathbf{M}_{\mathrm{III}}^{\mathrm{i}}+\mathbf{M}_{\mathrm{I}}^{\mathrm{m}}
\end{array}\right]}_{\mathbf{A}} \mathbf{u}_{I J}=\underbrace{\left[\begin{array}{cc}
-\left(\mathbf{M}_{\mathrm{II}}^{\mathrm{a}}\right)^{\mathrm{T}} & \mathbf{0} \\
\mathbf{0} & -\mathbf{M}_{\mathrm{II}}^{\mathrm{m}}
\end{array}\right]}_{\mathbf{B}} \mathbf{u}_{i j}
$$

where $\mathbf{M}_{\mathrm{I}}^{\mathrm{a}}, \mathbf{M}_{\mathrm{II}}^{\mathrm{a}}$ and $\mathbf{M}_{\mathrm{III}}^{\mathrm{a}}$ denote the top-left, top-right and bottom-right $3 \times 3$ sub-matrix of $\mathbf{M}^{\mathbf{a}}$ respectively, and the same denotation rule is also used to $\mathbf{M}^{\mathrm{i}}$ and $\mathbf{M}^{\mathrm{m}}$. Both $\mathbf{A}$ and $\mathbf{B}$ are $6 \times 6$ matrices.

Table 2

Elastic properties of phases

\begin{tabular}{llr}
\hline & $E(\mathrm{MPa})$ & $f_{1}(\mathrm{MPa})$ \\
\hline Case I-1 & & 5.0 \\
Particle & 87,514 & 5.0 \\
Interface & 31,255 & 5.0 \\
Matrix & 31,255 & \\
Case I-2 & & 10.0 \\
Particle & 31,255 & 1.5 \\
Interface & 31,255 & 5.0 \\
Matrix & 31,255 & \\
Case I-3 & & \\
Particle & & \\
Interface & 87,514 & 10.0 \\
Matrix & 31,255 & 1.5 \\
\hline
\end{tabular}

a

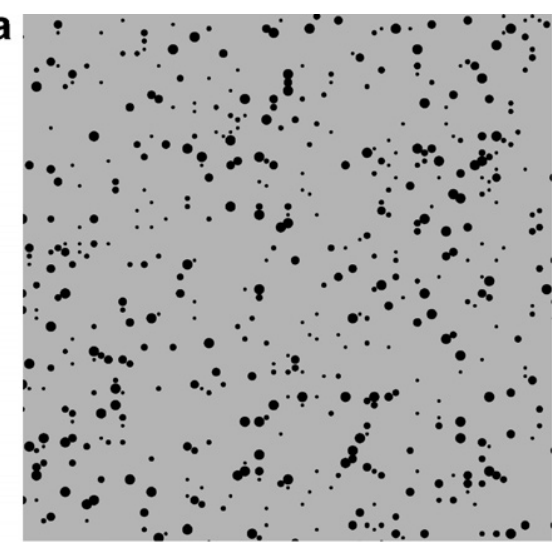

b

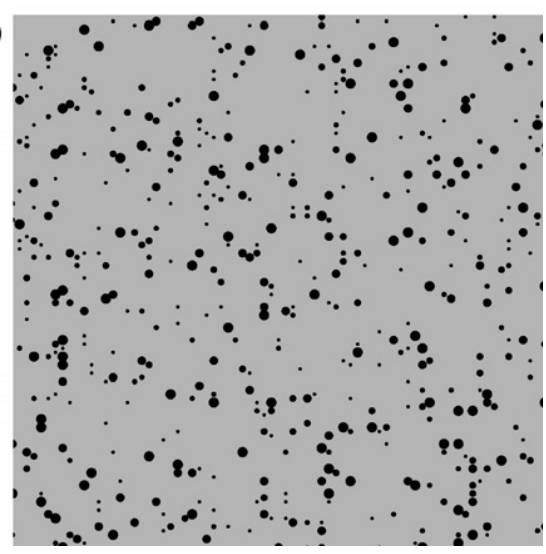

Fig. 7. The computer-generated particle overlay of (a) Case II-2; and (b) Case II-3. 
By solving the system of Eq. (17), the relationship between $\mathbf{u}_{I J}$ and $\mathbf{u}_{i j}$ can be expressed in the form

$$
\mathbf{u}_{I J}=\underbrace{\mathbf{A}^{-1} \mathbf{B}}_{R} \mathbf{u}_{i j}
$$

where $\mathbf{R}=\mathbf{A}^{-1} \mathbf{B}$.

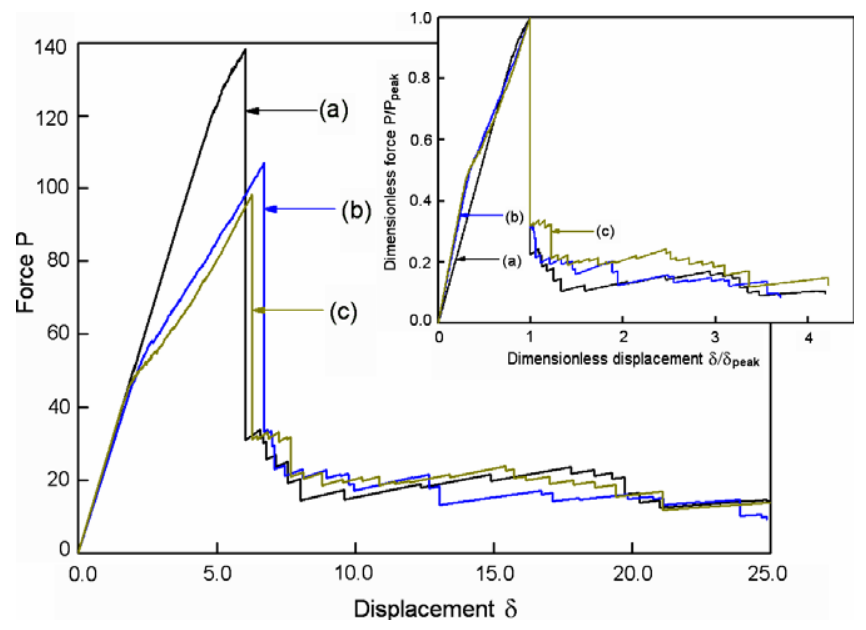

Fig. 8. $P-\delta$ curves for the cases in Group I: (a) Case I-1; (b) Case I-2; (c) Case I-3.
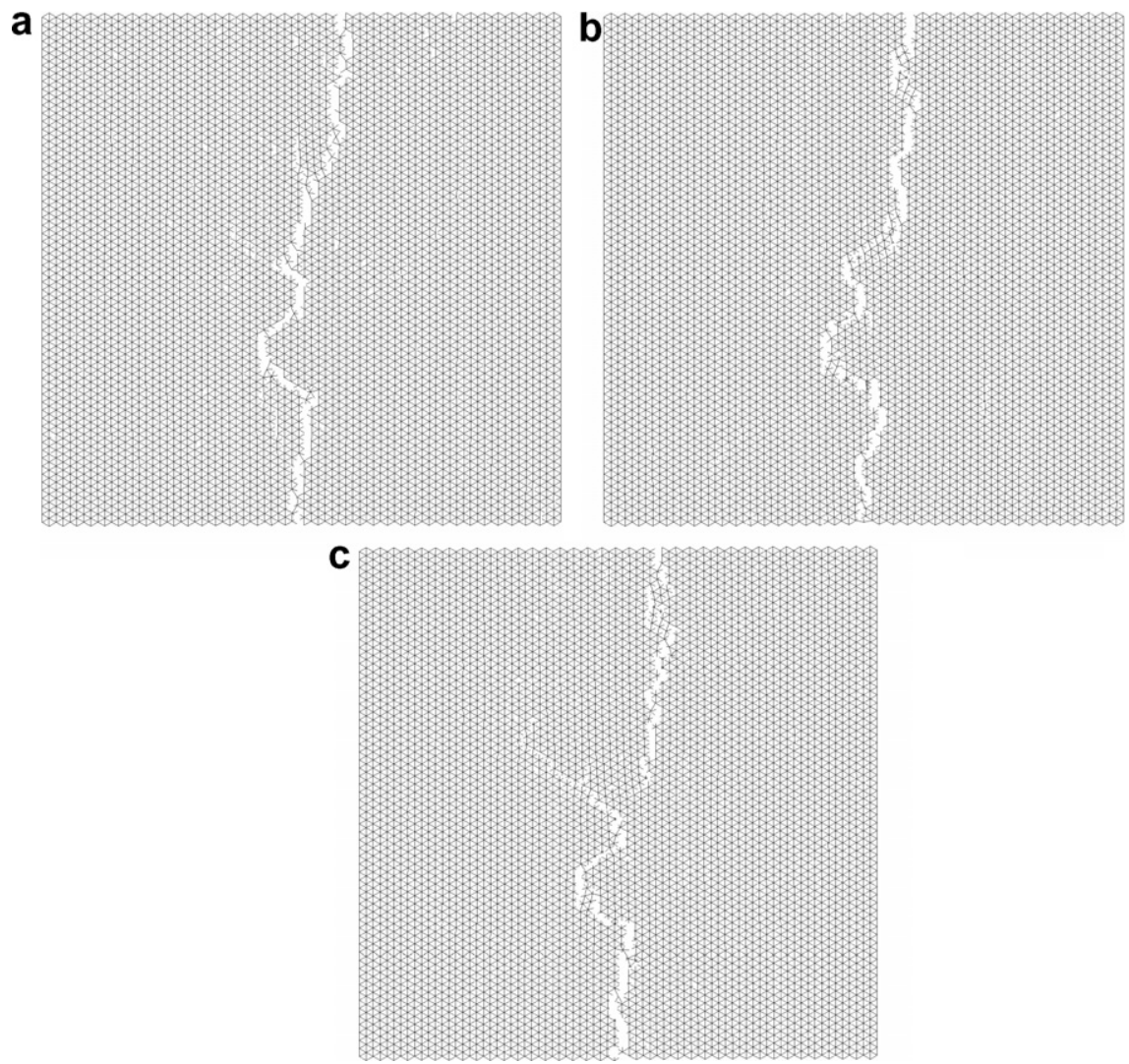

Fig. 9. Crack patterns directly following the peak-load for the cases in Group I: (a) Case I-1; (b) Case I-2; (c) Case I-3. 
$\mathbf{R}_{\mathrm{I}}, \mathbf{R}_{\mathrm{II}}$ and $\mathbf{R}_{\mathrm{III}}$ respectively denote the top-left, top-right, bottom-right $3 \times 3$ sub-matrix of $\mathbf{R}$. After a lengthy but elementary calculation, the stiffness matrix of the GB element $\mathbf{K}$ can be expressed in the form

$$
\mathbf{K}=\left[\begin{array}{cc}
\mathbf{M}_{\mathrm{I}}^{\mathrm{a}}+\mathbf{M}_{\mathrm{II}}^{\mathrm{a}} \mathbf{R}_{\mathrm{I}} & \mathbf{M}_{\mathrm{II}}^{\mathrm{a}} \mathbf{R}_{\mathrm{II}} \\
\mathbf{S Y M} & \left(\mathbf{M}_{\mathrm{II}}^{\mathrm{m}}\right)^{\mathrm{T}} \mathbf{R}_{\mathrm{III}}+\mathbf{M}_{\mathrm{III}}^{\mathrm{m}}
\end{array}\right]
$$

\section{The algorithm of solution}

As pointed out in the introduction, fracture is simulated by subsequent removal of critical elements from a lattice. Three beams in every GB element are judged whether to be critical independently. Once a beam approaches criticality, the corresponding GB element becomes a critical element. A criterion must be set to decide when a beam must be removed. In this paper the maximum tensile stress in each beam is computed in the following form [3]

$$
\sigma_{\text {eff }}=\frac{N}{A}+\alpha \frac{\left(\left|M_{i}\right|,\left|M_{j}\right|\right)_{\max }}{W}<f_{t}
$$

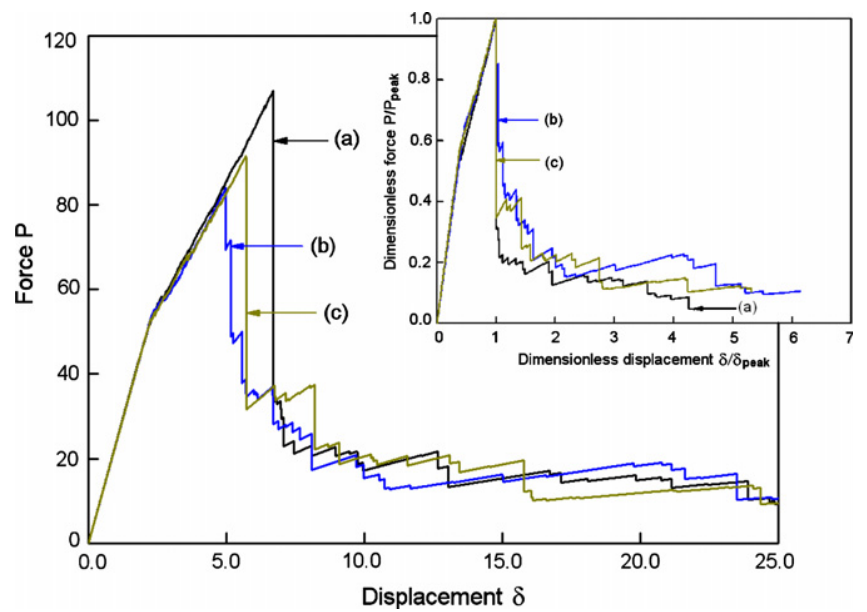

Fig. 10. $P-\delta$ curves for the cases in Group II: (a) Case II-1; (b) Case II-2; (c) Case II-3.

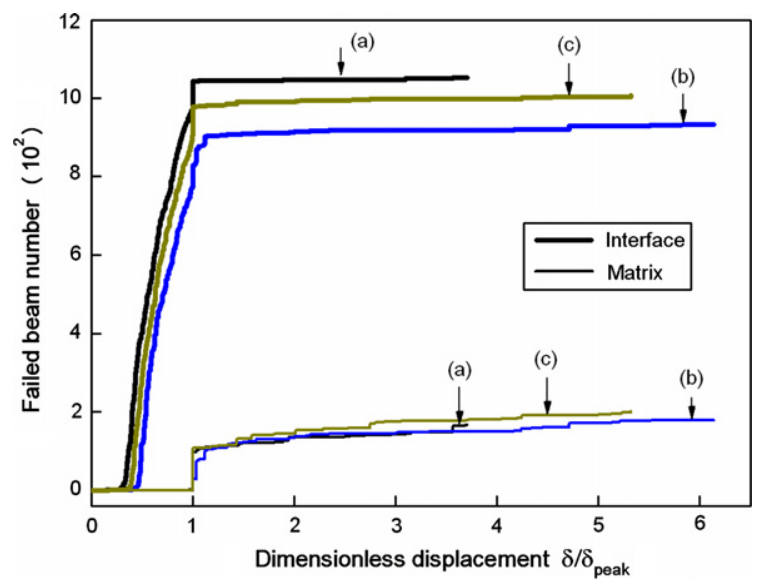

Fig. 11. Cumulative number of removed beams versus dimensionless displacement for the cases in Group II: (a) Case II-1; (b) Case II-2; (c) Case II-3. 
where $N$ is the normal force in the considered beam, $M_{i}$ and $M_{j}$ are the bending moments at the nodes $i$ and $j$ of the beam, and $W=t^{(b)} l^{2} / 6$ is the section modulus. The coefficient $\alpha$ regulates what part of the bending moment is considered. In this paper, $\alpha$ is $0.005[3,4,9,10]$. When the effective stress of a particular beam reaches its tensile strength $f_{t}$, brittle fracture is simulated by instantaneously removing the beam from the lattice.

\section{Numerical examples and discussions}

A concrete plate analyzed in $[3,4]$ is used as an example to investigate the effectiveness of the GB lattice in modeling the mechanical behavior of three-phase materials. Then the effects of the particle overlay on the fracture process are discussed in detail.

The plate is $(32 \sqrt{3} / 5) \times(32 \sqrt{3} / 5) \mathrm{cm}^{2}$. A regular triangular GB lattice having a total of $14234 \mathrm{~GB}$ elements and 4837 nodes is used in simulation. All elements are $\sqrt{3} / 10 \mathrm{~cm}$ long. The span of all interface beams is $\sqrt{3} / 100 \mathrm{~cm}$. The particle distribution is generated with the help of function " $r a n 0($ )" [16]. Randomly distributed particles with diameter $[0.4,1.2] \times \sqrt{3} / 10 \mathrm{~cm}$ are projected on to the GB lattice. The number of particles of each size is given in Table 1. All translational degrees of freedom are fixed along the left edge, while a uniform tension is applied to the right edge through a controlled displacement. In all the load-displacement curves, i.e. $P-\delta$ curves presented below, the units of $P$ and $\delta$ are $\mathrm{N}$ and $\mu \mathrm{m}$, respectively.

Two groups of numerical experiments are employed, which are denoted as Group I and Group II, respectively:

Group I. This group includes three cases: case I-1, case I-2 and case I-3. The three cases have a fixed particle overlay shown in Fig. 6, and different material properties in Table 2.

Group II. This group also includes three cases: case II-1, case II-2 and case II-3. The three cases have the same material properties as those of case I-2 in Table 2, and different particle overlays (Fig. 6 for case II-1, Fig. 7a for case II-2 and Fig. 7b for case II-3).

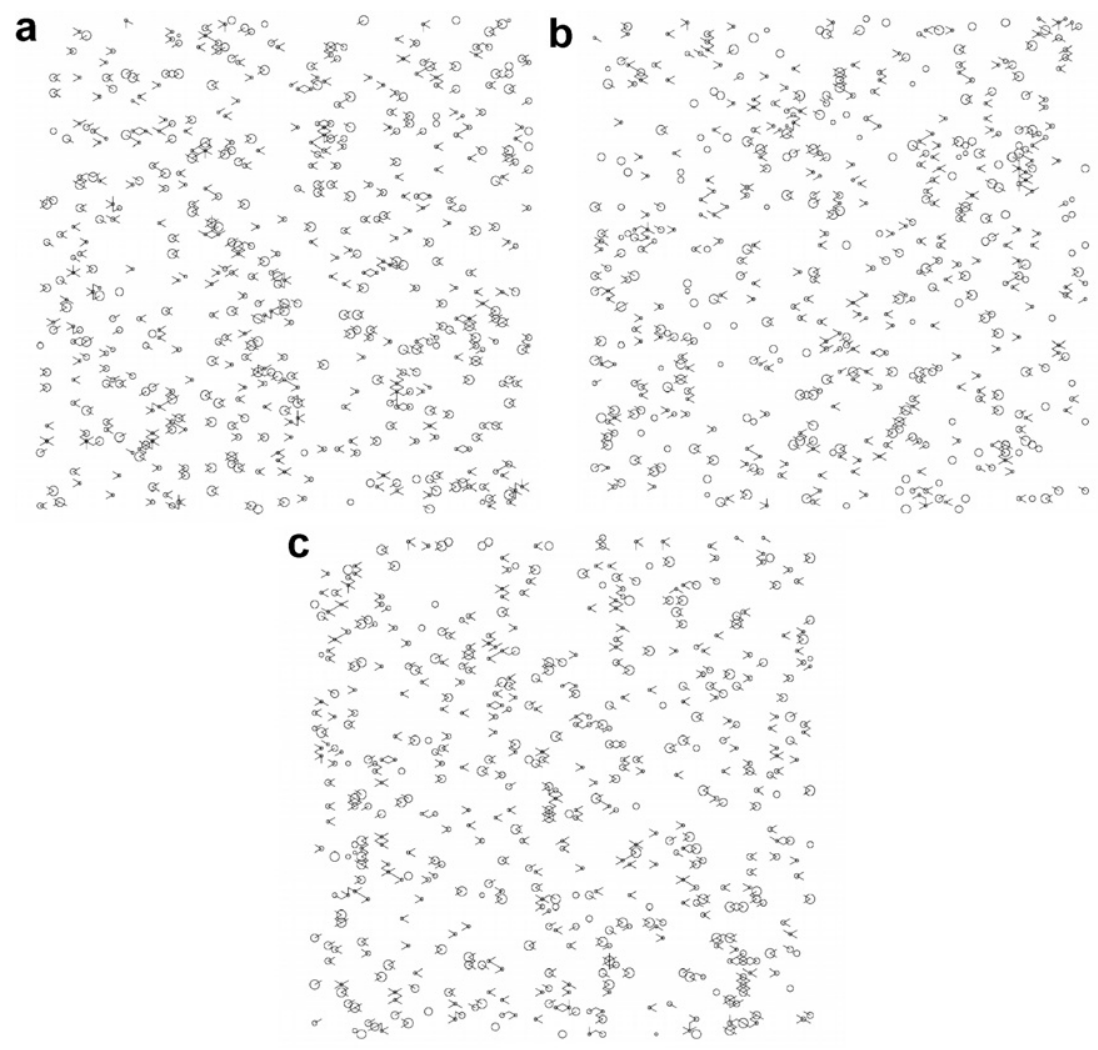

Fig. 12. Micro-cracking distributions at the peak-load level for the cases in Group II: (a) Case II-1; (b) Case II-2; (c) Case II-3. 


\subsection{Results}

Group I. The $P-\delta$ curves are shown in Fig. 8. The dimensionless $P-\delta$ curves, where load and displacement are divided by the values at the peak, are shown in the inset of Fig. 8. Crack patterns at load levels directly following peak-load levels are shown in Fig. 9.

Group II. The $P-\delta$ curves are shown in Fig. 10. The dimensionless $P-\delta$ curves are shown in the inset of Fig. 10. The number of failed beams versus dimensionless displacement is shown in Fig. 11. Micro-cracking distributions at the peak-load levels are shown in Fig. 12, where circles and short line-segments denote aggregates and failed elements, respectively. Crack patterns at levels directly following the peak-load levels are shown in Fig. 13.

\subsection{Analyses of a representative case}

The results of Group I and II have many features in common. Therefore, case II-3 is chosen as a representative. The dimensionless $P-\delta$ curve is shown in Fig. 14a. The number of failed beams versus displacement is shown in Fig. 14b. Crack patterns at typical load levels are shown in Fig. 15. Microscopic path of the macrocracks after the peak-load is shown in Fig. 16.

The $P-\delta$ curve in Fig. 14a shows two main branches, i.e. the pre-peak and the post-peak branch. In the beginning of the pre-peak regime, the specimen deforms elastically and no failure happens, leading to a linear elastic stage. After the first element has failed at the stage marked with a, the $P-\delta$ curve shows a kink. The 2D beam lattice cannot present the kink which has been observed noticeably in 3D simulations [10]. Then the part $a-b$ follows, which is nearly linear. Found in Fig. 14b, many interface beams fail subsequently during a-b. The peak-load appears at the level marked with $b$, and it is the dividing point between the pre-peak and the post-

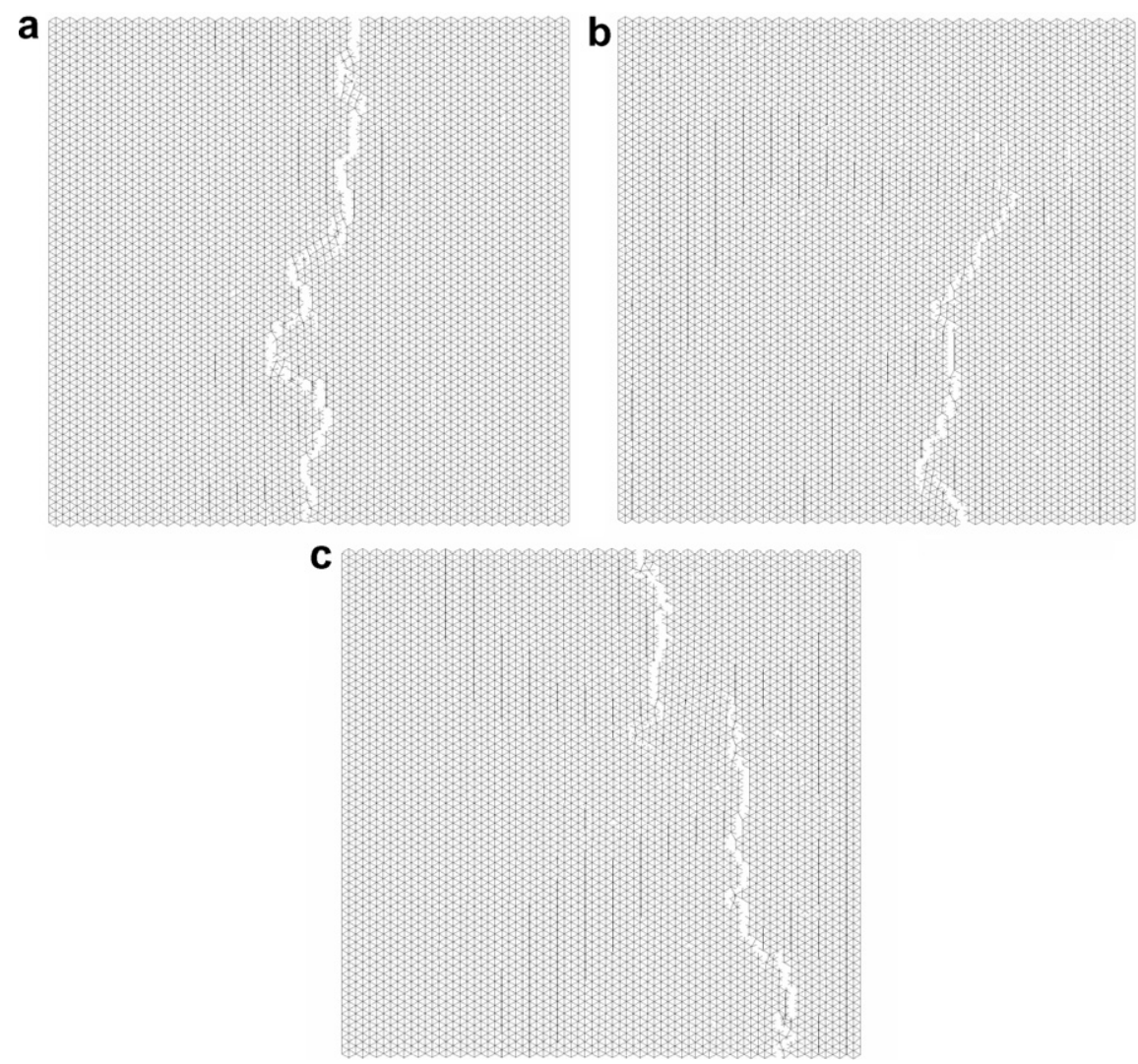

Fig. 13. Crack patterns directly following the peak-load for the cases in Group II: (a) Case II-1; (b) Case II-2; (c) Case II-3. 
a

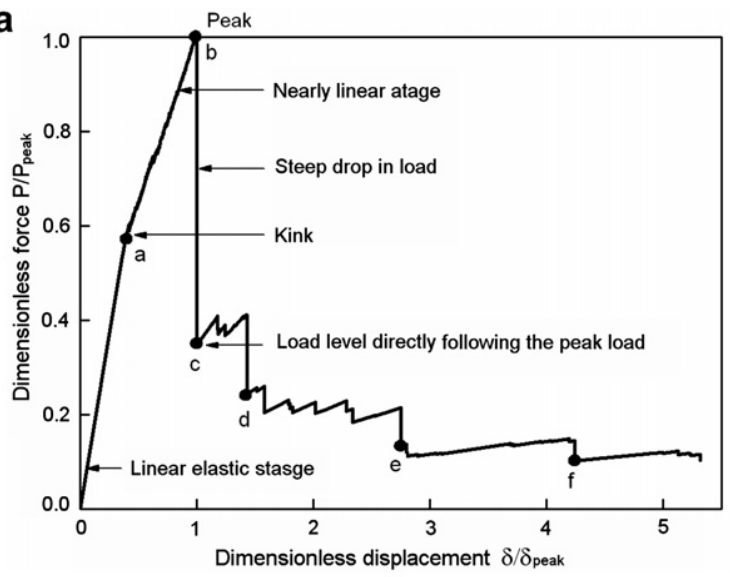

b

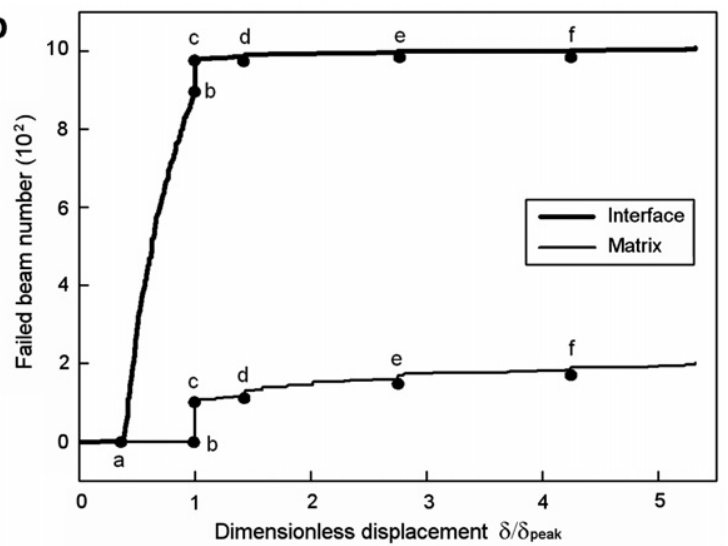

Fig. 14. For the representative case: (a) the dimensionless $P-\delta$ curve; (b) the cumulative number of removed beams versus dimensionless displacement.

peak branch. In 3D results [10], a strong nonlinearity can be found before the peak-load. However, this nonlinearity cannot be observed in Fig. 14a, which could be due to the exclusion of 3D effects. In the post-peak regime the curve shows subsequently a steep drop in load (until the stage marked with c) and a long tail.

Load varies stably with displacement in the pre-peak regime, but in the post-peak regime there are several steep drops in load (Fig. 14a). This can be explained as follows. The vast majority of fractured beams consist of interface beams in the pre-peak regime (Fig. 14b and Fig. 12c). Since stronger matrix beams are able to absorb the released stresses from the removed interface beams, micro-cracking happens here and there before nucleation of localized macro-crack (Fig. 12c). During b-c, two localized macro-cracks nucleate and extend, and part of the previously developed cracks is incorporated into the macro-cracks. Multiple macro-cracks were also observed experimentally [4,12]. The steep drops are associated to the sharp increase of number of fractured matrix beams (Fig. 14b). This means the macro-cracks mainly propagate in the matrix area (Fig. 16b) [10]. At the same time, the number of fractured interface elements also increases. The reason is that the weakest interface beams are not able to absorb the released stresses from the removed matrix beams. As a result, the fracture process shows high brittleness, leading to the steep drops in load. In the post-peak regime, the macro-crack extension is frequently deflected by aggregates, e.g. at point c, d, e and $\mathrm{f}$ (Figs. 15 and 16). Especially in Fig. 16b, at every turning in the macro-crack path, one or two aggregates can be found.

Additionally, aggregate beams fail during the post-peak process. It is questionable whether the fracture of aggregate beams can affect somehow the fracture process. However, only $3 \%$ of the total number of beams failed at the dimensionless displacement 5.32. This value is negligible when compared with the percentages of failed matrix and interface beams, which are $17 \%$ and $80 \%$, respectively [10]. 

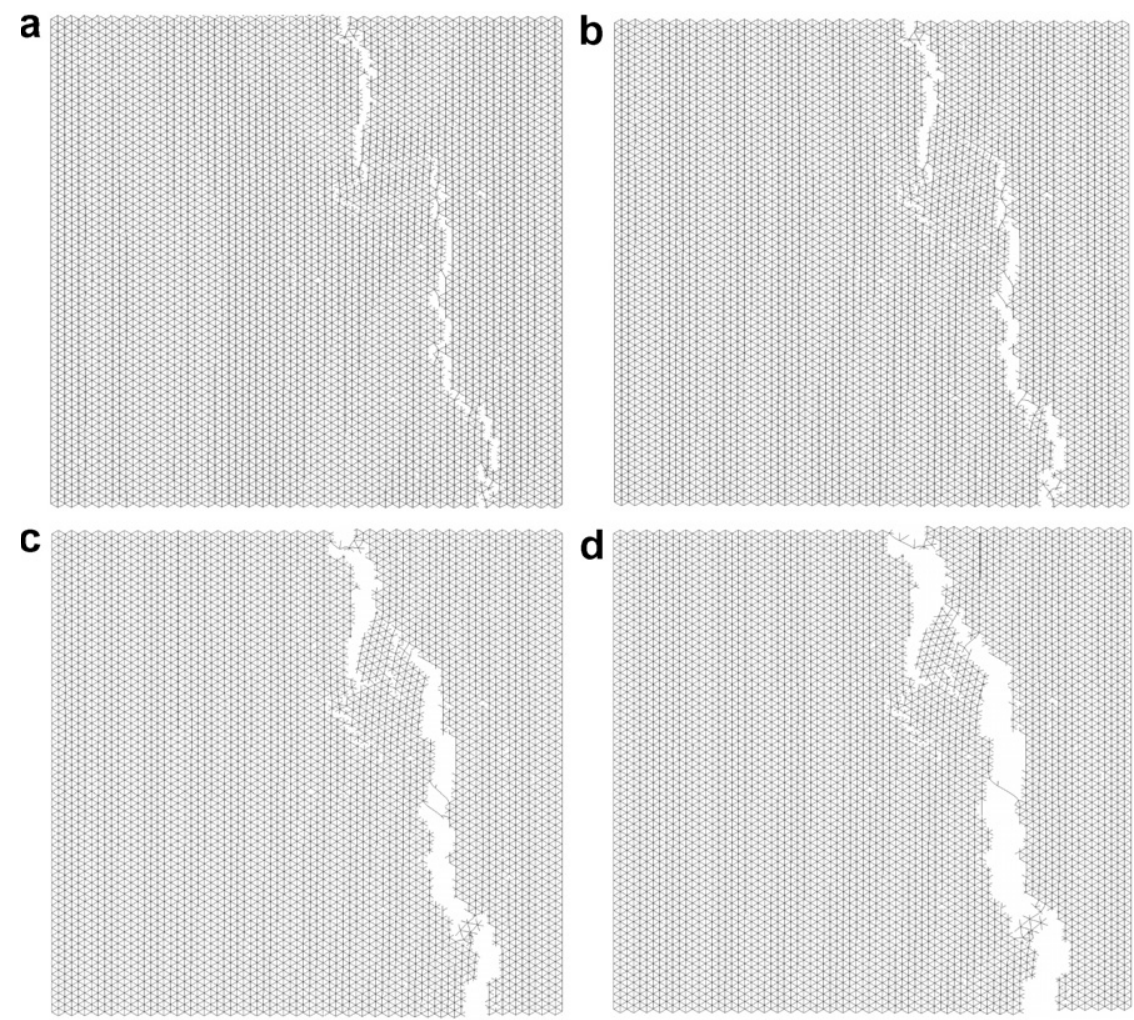

Fig. 15. Crack patterns at typical load levels for the representative case: (a) Point c; (b) Point d; (c) Point e; (d) Point f.
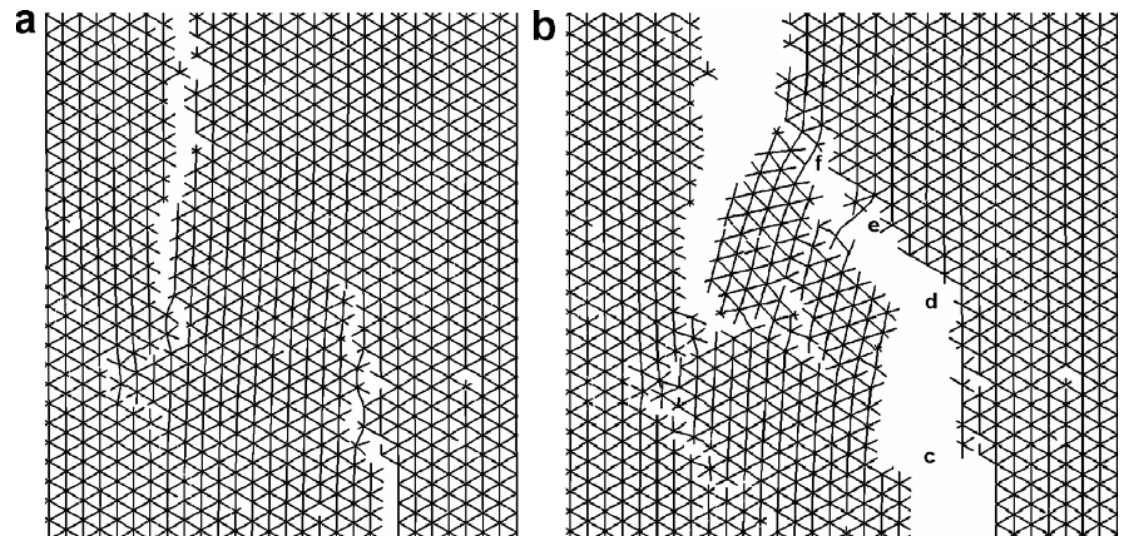

Fig. 16. Microscopic path of the localized macro-cracks for the representative case: (a) Point c; (b) Point f.

\subsection{Effects of the particle overlay on the fracture process}

The internal structure depends on the particle overlay. Because the macroscopic response is influenced by the material structure, it can be imagined that the particle overlay surely influences the behavior of the specimen. In this section, we dedicate to analyzing how the particle overlay influences the fracture process.

The first stage is the linear elastic stage before the first element has failed. The cases in Group I with the fixed particle overlay (Fig. 6) have different ending displacement values (Fig. 8). The reason for the difference is twofold: firstly, stress concentrations occur next to the particles due to the difference in stiffness among three 
phases; secondly, initial micro-cracking occurs in the beam with maximum value of the ratio between the effective stress and the tensile strength [10]. On the other hand, the cases in Group II have the same linear elastic stage approximately (Fig. 10). Therefore, the particle overlay has a negligible influence on this stage. Of course, the employed specimen should have so large a size that it can be the representative volume of the material. Otherwise, due to the expected boundary effect, the results in the linear elastic stage vary with the particle overlay [10].

The second stage is the nearly linear stage from the kink to the peak-load. From Fig. 10, the cases in Group II have the same slope approximately. Fig. 10 also shows a scatter of the peak-load. This scatter can be explained by the dissimilar distributions of the matrix areas where the macro-crack nucleates and extends. The peak-load level is the beginning of the localized crack [11].

Then the post-peak regime follows. As pointed out in the introduction, the ductility is associated with the post-peak behavior. The inset of Fig. 10 shows a noticeable scatter of ductility. This can be explained with the help of Fig. 11: in the post-peak regime, numbers of both fractured matrix and interface beams in the three cases have different tendencies with displacement, leading to the scatter of ductility.

Additionally, the crack pattern directly after the peak-load is analyzed. In Group I where the particle overlay is fixed, crack patterns (Fig. 9) are nearly uniform despite the difference of material properties. In Group II where the particle overlay varies, crack patterns (Fig. 13) are different from one another even though material properties are fixed. Obviously, the particle overlay plays a major role in the crack pattern.

On one hand, in the pre-peak regime, specimens of different particle overlays have nearly the same linear elastic stage, kink and slope of the nearly linear stage (Fig. 10). On the other hand, in the post-peak, their peak-loads, crack patterns and degrees of ductility are noticeably different (Fig. 10). By comparing Fig. 12 with Fig. 13, the macro-crack appears only after the peak-load. Therefore, nucleation and extension of the macro-crack are responsible for the scatter of the fracture process in the post-peak regime. As the discussion about Figs. 15,16 in Section 7.2, the path of the localized macro-crack depends on the internal structure, i.e. the particle overlay. In a word, variation of the particle overly can be the essential reason for the scatter of results under the present conditions.

\section{Conclusions}

To solve the problem of computational effort hampering the practical application of the beam lattice model a new lattice-type fracture model, i.e. the GB lattice model, was developed. The primary idea of the GB lattice model is the proposal of a kind of two-node and three-phase elements. In the beam lattice, an aggregate is simulated by a cluster of 10-100 nodes. In the GB lattice, however, an aggregate is modeled by just a single node. Therefore, much less computational effort is spent in the GB lattice model.

Numerical experiments were conducted on a panel subjected to uniaxial tension. The numerical results show that the model can reproduce the fracture process observed in real physical experiments.

Two groups of numerical experiments were employed to discuss the effects of the particle overlay on the fracture process. In Group I, where all the cases have a fixed particle overlay and different material properties, their $P-\delta$ curves are very different due to their different material properties, but their macro-crack paths are approximately the same. It indicates that the particle overlay plays a major role in the macro-crack path. In Group II, where all the cases have the same material properties and different particle overlays and the number of particles of each size is unchanged with the particle overlay, the specimens have nearly the same linear elastic stage, kink and slope of the nearly linear stage, but their peak-loads, crack patterns and degrees of ductility are noticeably different. Therefore, under the conditions of Group II, the particle overlay has a negligible effect on the pre-peak behavior, but a dominant effect on the post-peak behavior.

\section{Acknowledgements}

This research work was sponsored by National Natural Science Foundation of China through Grant No. 10572140 and No. 10232050, and also partly by Ministry of Science and Technology Foundation No. 2002CB412706. The first author, Jinxing Liu, is grateful to Wei Wu, Nan Liu, Xiaoliang Chen, Jiuan Wei, Yinchun Wang and Qiang $\mathrm{Fu}$ for their valuable discussions and suggestions. 


\section{References}

[1] H.M. Jennings, A model for the microstructure of calcium silicate hydrates in cement paste, Cement Concrete Res. 30 (2000) $101-116$.

[2] S. Mindess, Interfaces in concrete, in: J.P. Skalny (Ed.), Materials Science of Concrete, American Ceramic Society, Westerville, OH, 1989, pp. 163-180.

[3] J.G.M. van Mier, M.R.A. van Vliet, T.K. Wang, Fracture mechanisms in particle composites: statistical aspects in lattice type analysis, Mech. Mater. 34 (2002) 705-724.

[4] B.L. Karihaloo, P.F. Shao, Q.Z. Xiao, Lattice modelling of the failure of particle composites, Engng. Fract. Mech. 70 (2003) 23852406.

[5] T.K. Wang, J.G.M. van Mier, T.N. Bittencourt, Statistical study of fracture in concrete, in: K. Ravi-Chandar, B.L. Karihaloo, T. Kishi, R.O. Rithie, A.T. YokoboriJr, T. Yokobori (Eds.), Advances in Fracture Research. Proc ICF10 0665OR, Pergamon, 2001.

[6] J.E. Bolander, S. Saito, Fracture analyses using spring networks with random geometry, Engng. Fract. Mech. 61 (1998) 569-591.

[7] M. Ostoja-Starzewski, Lattice models in micromechanics, Appl. Mech. Rev. 55 (2002) 35-60.

[8] H.J. Herrmann, Patterns and scaling in fracture, in: J.G.M. van Mier, J.G. Rots, A. Bakker (Eds.), Fracture Processes in Concrete, Rock and Ceramics, vol. 1, E\&FN SPON, 1991, pp. 195-211.

[9] J.G.M. van Mier, B.M. Chiaia, A. Vervuurt, Numerical simulation of chaotic and self-organizing damage in brittle disordered materials, Comput. Meth. Appl. Mech. Engng. 142 (1997) 189-201.

[10] G. Lilliu, J.G.M. van Mier, 3D lattice type fracture model for concrete, Engng. Fract. Mech. 70 (2003) 927-941.

[11] J.G.M. van Mier, M.B. Nooru-Mohamed, Geometrical and structural aspects of concrete fracture, Engng. Fract. Mech. 35 (4-5) (1990) 617-628.

[12] E.P. Prado, J.G.M. van Mier, Effect of particle structure on mode I fracture process in concrete, Engng. Fract. Mech. 70 (2003) $1793-$ 1807.

[13] M. Ostoja-Starzewski, P.Y. Sheng, K. Alzebdeh, Spring network models in elasticity and fracture of composites and polycrystals, Comput. Mater. Sci. 7 (1996) 82-93.

[14] T.J.R. Hughes, R.L. Taylor, S. Kanoknukulchai, A simple and efficient finite element for bending, Int. J. Numer. Engng. 11 (1977) 1529-1543.

[15] X.C. Wang, Finite Element Method, first ed., Tsinghua, 2003, p. 309-315.

[16] Sample page from NUMERICAL RECIPES IN FORTRAN 77: THE ART OF SCIENTIFIC COMPUTING. Cambridge University Press, 1986-1992, pp. 267-272. 